\title{
Review Article \\ Modeling Requirements for Computer Simulation of Cerebral Aneurysm
}

\author{
S. R. Ghodsi, ${ }^{1}$ V. Esfahanian, ${ }^{2}$ and S. M. Ghodsi ${ }^{3}$ \\ ${ }^{1}$ VFE Research Institute, University of Tehran (Campus 2), College of Engineering, 4th Floor of Institute of \\ Petroleum Engineering Building, North Kargar Avenue, Tehran 14399-56191, Iran \\ ${ }^{2}$ School of Mechanical Engineering, University of Tehran, Tehran 14399-56191, Iran \\ ${ }^{3}$ Sina Trauma Research Center, Tehran University of Medical Science, Tehran 14399-56191, Iran \\ Correspondence should be addressed to S. R. Ghodsi; dr.sr.ghodsi@ut.ac.ir
}

Received 20 November 2013; Revised 8 January 2014; Accepted 8 January 2014; Published 16 February 2014

Academic Editor: Michele Migliore

Copyright @ 2014 S. R. Ghodsi et al. This is an open access article distributed under the Creative Commons Attribution License, which permits unrestricted use, distribution, and reproduction in any medium, provided the original work is properly cited.

Background. In order to reduce the mortality risk of aneurysm rupture, a timely diagnosis and treatment are vital. There are different reasons for aneurysm, such as hypertension, arteriosclerosis, and heredity. An efficient and cost-effective method to study the generation, development, and rupture of aneurysm and also analysis of treatment methods can accelerate progress. The Computational Fluid Dynamics is a well-known tool to simulate various phenomena. A reliable virtual modeling in biology depends on our knowledge about variety of characteristics, that is, biological features, structural properties, and flow conditions. Objective. Because of the vast research about the related subjects, an organized review is required. The aim of current review article is classification of the required foundations for a reliable virtual modeling of cerebral aneurysm, especially in the Circle of Willis.

\section{Introduction}

Up to $50 \%$ cases of Subarachnoid Hemorrhage (SAH) result in fatality and $10 \%-15 \%$ lead to death before reaching a hospital [1]. Rupture of cerebral aneurysm is a well-known cause of SAH. The most prevalent location of cerebral aneurysm is Circle of Willis (CoW). Different factors can increase the risk of aneurysm generation and development, for instance aging, arteriosclerosis, and heredity. In addition, the characteristics of blood flow can exacerbate the problem. Thus it is required to gather various clinical and engineering aspects to reach an actual understanding of the nature of aneurysm.

An efficient and cost-effective way to modeling natural phenomena is numerical simulation. Increasing power of computing causes numerical simulation that plays a key role in recognition of various problems in engineering and medicine. The important point is the reliability of obtained results and the meaningful results of a numerical simulation that depend on variety of aspects.

The classification of related subjects to numerical simulation is a valuable step. Because of the complexity of blood supply system in the brain and also long-time history of related research, it is necessary to review these studies. Two main groups are considered here: (1) fluid and structure and (2) flow and simulation. The tree of this classification is shown in Figure 1.

In fluid and structure section, there are three subcategories: blood properties, vessel properties, and cerebrovascular features. On the other side, the flow and simulation is divided to three subcategories: geometry and computational domain, numerical approaches, and flow regime features. The $\mathrm{CoW}$ is a collection of major vessels connected together and located at the bottom of the cerebral cavity and it is a common place for aneurysm [2]. The CoW combines the blood flow from the incoming afferent vessels and also transfers from a hemisphere to another [3]. The responsibility of CoW is compensating reduction of cerebral perfusion pressure, for example, when complete occlusion occurs in the internal carotid artery [4]. Some important physiological properties of CoW's vessels are presented in Table 1.

It was evident that the risk of transient ischemic attack is lower in the patients with healthy collateral circulations [5]. Approximately, at least $40 \%-50 \%$ of the people are confronted with the CoW's anomalies. The anomalies can be 


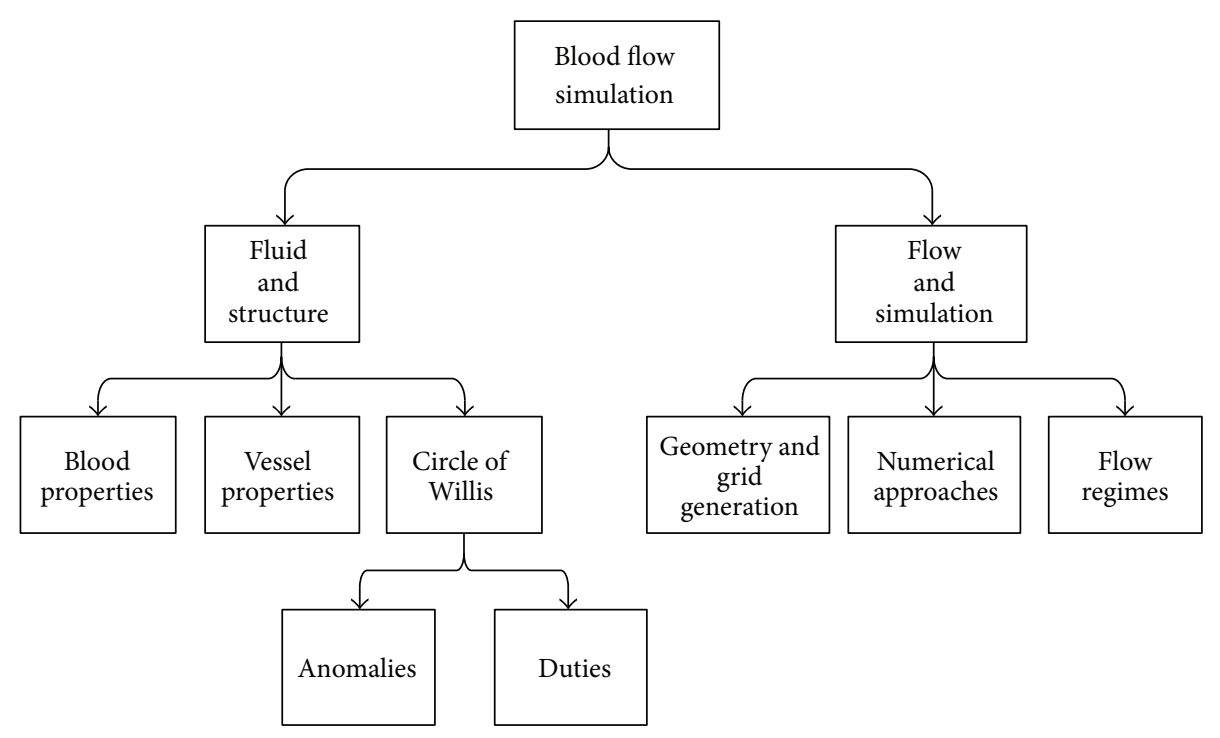

FIGURE 1: Classification of related subjects to the numerical simulation of blood flow in CoW.

classified in three cases: lack of some vessels, asymmetric, and different diameter of similar vessels.

The variations of CoW usually affect more than one segment. Papantchev et al. studied six types of common variations and their effects [6]. In order to know the condition of abnormality in Iranian population, 102 Iranian males were examined and classified in to the 22 common types of anomalies [7]. Because of the importance of CoW's anomalies, similar studies were performed in different countries. The data of 250 Egyptian patients showed that $68.3 \%$ and $38.3 \%$ of CoW consisted of complete anterior and complete posterior parts, respectively [8].

\section{Required Data for Computation}

The vessels of human are classified in three groups by size: arteries, veins, and capillaries. The artery and vein consist of three and two layers, respectively. The three layers are Tunica media, Tunica intima, and Tunica adventitia. Furthermore, the muscle layer in vein is considerably thinner than the artery's muscle layer [9].

Entire circulatory system is coated by endothelial cells. Low wall shear stress (less than $0.4 \mathrm{~Pa}$ ) causes the proliferation of endothelial cells and also atherosclerosis. On the other side, high wall shear stress (more than $1.5 \mathrm{~Pa}$ ) causes the reorientation of endothelial cells in the flow direction [10]. Some characteristics of vessels within the adult body are shown in Table 2. These data are important in computational domain generation. The complete data about other vessels and the variations are collected in the anatomy atlas [11]. The arterial bifurcation and atherosclerotic plaque can increase an abnormal shear stress on endothelium. The local arterial aggregate Young's Modulus of Elasticity decreases and subsequently causes an initial ballooning [12].

Obviously, the blood properties are required to simulate flow in arteries. The average adult has about 4 to 5 liters of blood. The red blood cells constitute about $45 \%$ of the volume of blood, and the remaining cells (white blood cells and platelets) less than $1 \%$. The fluid part of blood is called plasma and is about $55 \%$ [13].

The Reynolds number is an important flow criterion to identify the flow regime, that is, laminar or turbulent. Generally, the Reynolds number is the ratio of inertial forces to viscous forces. The Reynolds number is a dimensionless parameter

$$
\operatorname{Re}=\frac{\rho u L_{\mathrm{ref}}}{\mu}
$$

where $\rho, u, L_{\text {ref }}$, and $\mu$ are density, velocity, reference length, and viscosity, respectively. The reference length is a characteristic of geometry, for example, the vessel's diameter. The Reynolds number in most of the vessels is lower than 500, just in Aorta and Vena cave it is about 3400 . Thus, the blood flow is considered as laminar flow. However, under some conditions, for example, stenosis, the blood flow becomes turbulent in large diameter vessels, such as aorta [14].

If the Reynolds number is lower than the critical Reynolds, the flow is simulated as a laminar flow. The problem is finding the critical Reynolds number. In some cases the flow condition is on the border of turbulence and proper turbulent model is required [15]. The energy of blood flow decreases as a result of turbulence. Then the heart should add more energy to the blood flow. In addition, turbulence increases the perfusion pressure and also the required energy to overcome the additional resistance. In the microcirculation, including the arterioles, the linear relation between perfusion pressure and flow is

$$
P_{A}-P_{v}=q R_{p}
$$

where $P_{A}, P_{v}, q$, and $R_{p}$ are arterial pressure, venous pressure, flow rate, and peripheral resistance. The elasticity of the arterial network maintains high levels of perfusion pressure without overloading the heart, which is called the windkessel 
TABle 1: Physiological properties of CoW's vessels, such as length, radius, thickness, and elastic modulus [25].

\begin{tabular}{|c|c|c|c|c|c|}
\hline & Arteries & Length $(\mathrm{cm})$ & Initial diameter $(\mathrm{cm})$ & Thickness $(\mathrm{cm})$ & Elastic modulus $(\mathrm{Pa}) \times 10^{6}$ \\
\hline 1 & Ascending aorta & 4 & 2.4 & 0.163 & 0.4 \\
\hline 2 & Aortic arch I & 2 & 2.24 & 0.126 & 0.4 \\
\hline 3 & Brachiocephalic & 3.4 & 1.24 & 0.08 & 0.4 \\
\hline 4 & Aortic arch II & 3.9 & 2.14 & 0.115 & 0.4 \\
\hline 5 & L. common carotid & 20.8 & 0.5 & 0.063 & 0.4 \\
\hline 6 & R. common carotid & 17.7 & 0.5 & 0.063 & 0.4 \\
\hline 7 & R. subclavian & 3.4 & 0.846 & 0.067 & 0.4 \\
\hline 8 & Thoracic aorta & 15.6 & 2 & 0.11 & 0.4 \\
\hline 9 & L. subclavian & 3.4 & 0.846 & 0.067 & 0.4 \\
\hline 10 & L. ext. carotid & 17.7 & 0.3 & 0.038 & 0.8 \\
\hline 11 & L. int. carotid I & 17.7 & 0.4 & 0.05 & 0.8 \\
\hline 12 & R. int. carotid I & 17.7 & 0.4 & 0.05 & 0.8 \\
\hline 13 & R. ext. carotid & 17.7 & 0.3 & 0.038 & 0.8 \\
\hline 14 & R. vertebral & 14.8 & 0.272 & 0.034 & 0.8 \\
\hline 15 & R. brachial & 42.2 & 0.806 & 0.067 & 0.4 \\
\hline 16 & L. brachial & 42.2 & 0.806 & 0.067 & 0.4 \\
\hline 17 & L. vertebral & 14.8 & 0.272 & 0.034 & 0.8 \\
\hline 18 & L. int. carotid II & 0.5 & 0.4 & 0.05 & 1.6 \\
\hline 19 & L. PCoA & 1.5 & 0.146 & 0.018 & 1.6 \\
\hline 20 & R. PCoA & 1.5 & 0.146 & 0.018 & 1.6 \\
\hline 21 & R. int. carotid II & 0.5 & 0.4 & 0.05 & 1.6 \\
\hline 22 & Basilar & 2.9 & 0.324 & 0.04 & 1.6 \\
\hline 23 & L. MCA & 11.9 & 0.286 & 0.036 & 1.6 \\
\hline 24 & R. MCA & 11.9 & 0.286 & 0.036 & 1.6 \\
\hline 25 & L. ACA, A1 & 1.2 & 0.234 & 0.029 & 1.6 \\
\hline 26 & R. ACA, A1 & 1.2 & 0.234 & 0.029 & 1.6 \\
\hline 27 & L. PCA, P1 & 0.5 & 0.214 & 0.027 & 1.6 \\
\hline 28 & R. PCA, P1 & 0.5 & 0.214 & 0.027 & 1.6 \\
\hline 29 & L. ACA, A2 & 10.3 & 0.24 & 0.030 & 1.6 \\
\hline 30 & R. ACA, A2 & 10.3 & 0.24 & 0.030 & 1.6 \\
\hline 31 & $\mathrm{ACoA}$ & 0.3 & 0.148 & 0.019 & 1.6 \\
\hline 32 & L. PCA, P2 & 8.6 & 0.21 & 0.026 & 1.6 \\
\hline 33 & R. PCA, P2 & 8.6 & 0.21 & 0.026 & 1.6 \\
\hline
\end{tabular}

TABLE 2: The properties of blood vessels.

\begin{tabular}{lccc}
\hline Vessel & Number & Diameter $(\mathrm{cm})$ & $\mathrm{Re}_{\text {average }}$ \\
\hline Aorta & 1 & 2.5 & 3400 \\
Arteries & 159 & 0.4 & 500 \\
Arterioles & 400 & 0.005 & 0.7 \\
Capillaries & 4500 & 0.0008 & 0.002 \\
Venules & 4000 & 0.002 & 0.01 \\
Veins & 40 & 0.5 & 140 \\
Vena cava & 18 & 3 & 3300 \\
\hline
\end{tabular}

effect. The elastic function helps perfusion during diastole and generates the wave propagation [16].

The other main feature of fluid is viscosity. In the nonNewtonian fluid, there is a nonlinear relation between shear stress and shear rate. The non-Newtonian fluids are classified into the shear thinning and shear thickening. In shear thinning, increasing shear rate reduces the viscosity, and in shear thickening it is vice versa.

The human blood is considered as Newtonian fluid for all rates of shear when hematocrits are up to 12 percent. When the size of particles is significantly large in comparison with the channel's dimension, the fluid behaves like non-Newtonian fluid [9].

Consequently, in the large vessels such as arteries the blood is Newtonian, but in small vessels such as capillary, it is considered as non-Newtonian fluid. The effect of vessel's diameter on the viscosity is called Fahraeus-Lindqvist. As the diameter decreases (between 10 and 300 micrometers), the blood viscosity is reduced, because of the motion of erythrocytes in the center of the vessel and leaving plasma near the wall. A comprehensive study on different viscosity models of blood was performed by Yilmaz and Gundogdu. The models are classified into two groups including time independent 
and time dependent flow behavior models [17]. The impact of viscosity models on the simulation of aneurysm is studied by Evju et al. [18]. It has been shown that the nonlinear viscosity model can be important in some cases of aneurysm.

The shear forces due to the viscosity rises the pressure drop in network of vessels. Generally, the Hagen-Poiseuille equation is used to compute pressure drop of a laminar and incompressible flow in long cylindrical pipe:

$$
\Delta p=\frac{8 \mu L Q}{\pi r^{4}}
$$

where $L, Q$, and $r$ are pipe's length, volumetric flow rate, and pipe's radius, respectively. The Hagen-Poiseuille equation is just used for a Newtonian fluid. In capillary vessel, the crosssection can be divided into two regions including a core and a cell-free plasma region near the wall. So, if the plasma layer thickness is $\delta$, then

$$
\begin{aligned}
& \text { Core Region }\left(0 \leq r \leq r_{\text {cap }}-\delta\right) \\
& \qquad-\frac{\Delta p}{L}=\frac{1}{r} \frac{d}{d r}\left(\mu_{c} r \frac{d u_{c}}{d r}\right) \\
& \text { Cell-free plasma }\left(r_{\text {cap }}-\delta \leq r \leq r_{\text {cap }}\right) \\
& -\frac{\Delta p}{L}=\frac{1}{r} \frac{d}{d r}\left(\mu_{p} r \frac{d u_{p}}{d r}\right),
\end{aligned}
$$

where $u_{c}, \mu_{c}, u_{p}$, and $\mu_{p}$ are velocity and viscosity in core and cell-free regions, respectively. Some assumptions are required to use these equations including no slip condition, zerogradient in the center of vessel, and continuous velocity and shear stress at the regions' interface.

If an oscillating pressure gradient exists in the flow field, the velocity profile is a parabolic and proportional to the instantaneous flow rate and also the flow field is unsteady. Actually, in in vivo blood, the flow is affected by a pulsatile source. The inertia of the fluid in the central core prevents the core from following the applied gradient of pressure [19]. A dimensionless number, which is called Womersley, relates the frequency of unsteady pulsatile flow to viscous effect, in order to keep dynamic similarity in experimental scaling. The Womersley number implies how much of the vessel is affected by boundary layer, as follows:

$$
\alpha \propto \frac{\text { unsteady inertial force }}{\text { viscous force }} \longrightarrow \alpha=r_{\text {ves }} \sqrt{\frac{\omega \rho}{\mu}},
$$

where $\omega$ is the angular frequency of the oscillation, that is, the frequency of the heart rate [19]. Small Womersley number $(\alpha \leq 1)$ means the low frequency of pulsation. Therefore, parabolic profile of velocity is generated in each cycle and also Poiseuille's law is a good approximation [20]. On the other side, large number $(10 \leq \alpha)$ means flat velocity profile, and the mean flow delays the pressure gradient by $90^{\circ}$ [21].

Another important property of blood is density. Same as viscosity, not only the amount of density is not fixed for all cases, but also it is function of different variables, such as species, gender, and body posture. The density of human blood is approximately 1056 1060 $\left(\mathrm{kg} / \mathrm{m}^{3}\right)$. The density of blood plasma is approximately 1025 and $1125\left(\mathrm{~kg} / \mathrm{m}^{3}\right)$ [22].

The pressure gradient affording blood flow to the brain is called the Cerebral Perfusion Pressure (CPP). The variation of CPP is limited, because it can cause brain tissue to become ischemic, or rising Intracranial Pressure (ICP) [23]. The CPP is related to the cerebral blood flow (CBF) by the CerebroVascular Resistance (CVR) as follows:

$$
\mathrm{CBF}=\frac{\mathrm{CPP}}{\mathrm{CVR}}
$$

The autoregulation is a significant ability of human body to regulate the proper perfusion pressure for sensitive and vital organs. The brain arteries can respond to the perfusion pressure change by vasodilate or vasoconstrict, which is called autoregulation.

The autoregulation mechanism consists of two features, the autoregulation curve and the autoregulation dynamics [24]. In the autoregulation curve, the autoregulated range of pressure is approximately $75-175 \mathrm{mmHg}$ and the overall steady-state blood flow rate is a function of mean arterial blood pressure:

$$
Q_{\mathrm{ss}}=\frac{1}{1+A\left[\mathrm{O}_{2}\right]_{A}}\left(M A+\frac{p_{A}-p_{v}}{R_{0}}\right)
$$

where $Q_{s s},\left[\mathrm{O}_{2}\right]_{A}, M, p_{A}$, and $p_{v}$ are the steady-state blood flow rate, arterial oxygen concentration, metabolic rate, arterial pressure, and venous pressure, respectively. The $A$ and $R_{0}$ are two parameters to fit the autoregulation curve.

The upper and lower limits of the permeability are related to the arterial pressures limits of the autoregulated range. Therefore, the autoregulation dynamics are modeled using permeability $(k)$ as follows:

$$
\tau \frac{d k}{d x}=K_{p} \frac{Q_{s s}-Q}{Q_{s s}}+K_{i} \int_{0}^{t} \frac{Q_{s s}-Q}{Q_{s s}} d t
$$

where $Q$ is time-varying flow rate and $\tau, K_{p}$, and $K_{i}$ are time constant, proportional, and integral gains, which are considered as 3, 70, and 0.1, respectively [24].

\section{Simulation and Modeling Methods}

The cerebral blood flow is a complex system, and CoW is just one of the important parts. Therefore, the systematic analysis is necessary for recognizing the functionality of CoW in this dynamic system. From the numerical modeling view point, there are three models of arterial blood flow [16].

3.1. Zero-Dimensional (OD) Models. The lumped element model describes the behavior of a distributed system into discrete entities under certain assumptions. The Windkesselmodel of the arterial network reveals a relation between 
pressure and flow at a specific arterial condition, without wave propagation consideration.

3.2. One-Dimensional (1D) Models. The simplified equations of fluid motion are used to study the flow field. The wavetransmission characteristics are formulated by Womersley's oscillatory flow theory because of the hyperbolic feature of equations. A comprehensive literature review on the $1 \mathrm{D}$ models was performed by Reymond et al. [26].

3.3. Three-Dimensional (3D) Models. In order to simulate accurately a segment of arterial tree, more comprehensive 3D model is required. Computational Fluid Dynamic (CFD) is a powerful tool to simulate complex flow field. The $0 \mathrm{D}$ and $1 \mathrm{D}$ modeling can reveal some useful and practical aspects about a real complex system. In order to investigate a phenomenon in detail, more accurate and applicable methods are required. If whole system of cerebral vessels is simulated by an accurate three-dimensional approach, the computational cost is beyond the power of current computing devices. The studies based on CFD are classified into three: the development, progression, and rupture of cerebral aneurysms [27]. The experiences show that image-based CFD is a capable facility to study interaction between hemodynamic loading and mechanobiological wall responses.

Combination of above methods can help improve prediction of complex problems. Quarteroni and Veneziani coupled 3D modeling of blood flow with a systemic, zerodimensional, lumped model of circulation [28]. Beulen et al. derived boundary conditions in the first period from a onedimensional wave propagation model and the initial pressure distribution [29].

One of the earliest studies about the mathematical model of saccular aneurysm in CoW was performed by Austin in 1971 [30]. The intracranial aneurysm was simulated using electrical circuit and also Duffing equation. He studied on the modeling of the effect of flow frequencies range on the flow condition in saccular aneurysm. This study was extended by Cronin to show the importance of pulsatile pressure in the danger of aneurysm rupture [31].

Cassot et al. developed a mathematical model to simulate the CoW flow and predict the effectiveness of anterior and posterior communicating arteries on the brain blood pressure [32]. This model was based on a linear function of the mean blood flow in 10 bifurcations in CoW and the difference of the pressures at the extremities of these nodes. After a decade, Lorthois et al. presented the modified and advanced 1D nonlinear model using complex rheological properties of blood flow (such as and hematocrit distributions) in microcirculation $[33,34]$.

The equations of $1 \mathrm{D}$ models are based on the governing equations of flow motion. The laminar flow in arteries is same as steady and fully developed Poiseuille flow, therefore, the axial pressure gradient is computed as [35]

$$
\frac{\partial p}{\partial z}=-\frac{8 \pi \mu u}{A} .
$$

In order to make conservation form, the equations are coupled in a system of equations as

$$
\frac{\partial}{\partial t}\left[\begin{array}{l}
A \\
u
\end{array}\right]+\frac{\partial}{\partial z}\left[\begin{array}{c}
u A \\
\frac{u^{2}}{2}+\frac{p}{\rho}
\end{array}\right]=\left[\begin{array}{c}
0 \\
-\frac{8 \pi \mu}{\rho} \frac{u}{A}
\end{array}\right] .
$$

There are three variables in this system of equations, and an additional equation is needed to solve them. The third equation describes the relation between variation of artery's area and pressure (i.e., fluid-structure interaction). Therefore, the Laplace law for thin and homogenous elastic artery's wall is used to make this equation as follows:

$$
p-p_{0}=\beta\left(\sqrt{A}-\sqrt{A_{0}}\right), \quad \beta=\frac{E h_{0} \sqrt{\pi}}{A_{0}\left(1-\sigma^{2}\right)},
$$

where $A_{0}$ and $h_{0}$ are the artery's area and wall thickness at the reference state. In addition, $\sigma$ and $E$ are Poisson's ratio and Young's Modulus.

The one-dimensional model can be deduced from the wave propagation relation. The average speed of blood in vessels is $0.5 \mathrm{~m} / \mathrm{s}$, but the wave speed is $5 \mathrm{~m} / \mathrm{s}$. The blood pressure and flow pulsations are considered as the wave propagation in the arterial network, which consist of information about the cardiovascular system [16].

An important factor in the instantaneous vessel's area is pressure difference between two sides of a vessel's wall, which is called transmural pressure $\left(p_{\mathrm{tr}}\right)$. There is a nonlinear and frequency relation between the transmural pressure and the area of the vessel, which is caused by complex nonlinearity and viscoelasticity of the arterial wall. The wave propagation speed of blood was introduced by Young as

$$
c=\sqrt{\frac{A}{\rho C}}=\sqrt{\frac{E h}{2 \rho a}},
$$

where $C$ is local area compliance. This equation is derived from simplified Newton's second law of motion and it is called Moens-Korteweg equation model.

Generally, the characteristic lines are defined in hyperbolic equations and they determine the behavior of solutions using invariant quantities along certain trajectories. The conservative form of hyperbolic system of equations appears as [35]

$$
\begin{gathered}
\frac{\partial U}{\partial t}+H \frac{\partial U}{\partial x}=C \\
U=\left[\begin{array}{l}
A \\
u
\end{array}\right], \quad H=\left[\begin{array}{cc}
u & A \\
\frac{\beta}{2 \rho \sqrt{A}} & u
\end{array}\right], \\
C=\left[-\frac{1}{\rho}\left(\frac{8 \pi \mu u}{A}+\frac{\partial p_{0}}{\partial x}-\frac{\beta}{2 \sqrt{A_{0}}} \frac{\partial A_{0}}{\partial x}+\left(\sqrt{A}-\sqrt{A_{0}}\right) \frac{\partial \beta}{\partial x}\right)\right] .
\end{gathered}
$$

The speed of waves from the heart (forward) is $u+c$ and also towards the heart (backward) is $u-c$. The forwardrunning wave is generated by the contraction of the heart 
and moves through the arterial network. When there is a variation in vessel properties, such as branch, the wave is reflected and moves back towards the heart. Therefore, there is a combination of forward- and backward-running waves.

In order to solve this system, the boundary conditions are required. The boundary conditions are reflecting and nonreflecting. In nonreflecting boundary conditions, the forward and backward waves are used at the inlet and outlet boundaries, respectively. Thus, the information from inside and outside of the domain are combined.

It should be noted that there are some difficulties in solution, such as discontinuity. A discontinuity is considered as a sudden jump; for example, stent or in a more prevalent case it is a vessel branching.

In the brain, aneurysms are often located at the lateral sides of curved vessels and at the bifurcations. The ruptured aneurysm is a common reason of death and also the surgery of aneurysm is high risk. Therefore, the recognition of different related subjects can improve the prediction of its behaviors. Different scientists studied the subjects, such as the reasons of aneurysm, the growth trend, and the effective factors. The effect of hemodynamic factors on the initiation, growth, and rupture of aneurysms is shown in different studies [36-39]. Furthermore, it is observed that the CFD-based prediction of aneurysm rupture is gradually becoming more confident and reliable [40].

Furthermore, the interaction between blood and vessel's wall play crucial role to simulate semireal pattern of fluid flow. The pressure and also blood velocity in large arteries are affected by the vessel deformability. The fluid-solid interaction (FSI) methods are used to predict this interaction. In simulation of a real situation, if the wall tension exceeds the wall tissue strength, the rupture of tissue should occur [41]. Furthermore, accuracy of modeling the arterial endothelial dysfunction plays an important role in prediction of long term vessel deformation [42]. ods:

Generally, there are some common points in FSI meth-

(1) generated loads on the structure by fluid,

(2) response of structure and impacts on the flow field,

(3) computational domain and grid adaptation according to new conditions.

Based on significant development in computational fluid approaches and FSI methods and also advanced software to generate complex computational domain from in vivo medical imaging, the prediction of aneurysm behavior become more accurate and reliable [15, 43, 44]. Figueroa et al. developed a method in order to simulate blood flow in deformable models of arteries. They coupled the equations of the deformation of the vessel wall as a boundary condition for the fluid zone [45].

One of the first successful attempts to use FSI in predicting the abdominal aortic aneurysm was performed by Di Martino et al. [46]. The risk of rupture is a function of transversal diameter, that is, the maximum safe crosssectional area of aneurysm. The aneurysm is usually asymmetric; therefore, the simple method, such as Laplace law is not an appropriate solution of this question. An accurate 3D FSI simulation can predict correct relation between the growth rate of aneurysm and flow conditions. Li and Kleinstreuer used FSI simulation to investigate the geometry variations of abdominal aortic aneurysm, such as degree of asymmetry, neck angle, and bifurcation angle [47].

The functionalities and anomalies of CoW are studied using CFD tools [48-51]. Alnæs et al. developed a 3D simulation of CoW to study the impact of variations in vessel radii and bifurcation angles on wall shear stress and pressure on vessel walls [52]. It is shown that the rigid vascular wall is a good assumption for smaller vessels but not for larger arteries. In the CoW, this approximation could overestimate wall shear stress but probably not influence its spatial distribution.

Kim simulated the flow condition of CoW in two cases, including absence of left PCoA and absence of ACoA. It was shown that the autoregulation mechanism is strongly affected by the communicating arteries, PCoA and ACoA [53]. Long et al. studied on the CoW functionalities and abilities to supply blood in patients with unilateral carotid arterial stenosis. They selected four CoW configurations with an axisymmetric stenosis in an internal carotid artery (ICA) and also different boundary conditions [51].

In spite of these significant abilities, there are some difficulties to reach accurate and applicable predictions:

(1) geometry generation according to the real situation,

(2) generation of proper and efficient computational grid,

(3) appropriate and efficient solver selection,

(4) accurate initial and boundary conditions,

(5) trace the result during the run carefully to improve the simulation,

(6) present the final results in a user-friendly and inferable manner.

The most important subject in geometry generation is similarity with the physical domain. The simplest numerical form of aneurysm is a spherical and symmetric sac. But the actual geometry of aneurysms is irregular. Some software can generate real geometry from X-ray scans. For example, the mimiced software can produce actual geometry of organs from MRI of CT-scan files [54]. These tools can help reduce significantly the time of preprocessing and also improve the final results.

After the geometry and grid generation, the initial and boundary conditions are required. Generally, the boundary value problem is a differential equation with boundary conditions. The boundary conditions cause unique solution for problem, which is called well-posed problem. In addition, the relation between computational domain and outer regions is established with boundary condition. The boundary condition types are inlet, outlet, wall, symmetry, and periodic. Another important factor is initial condition, which specifies the condition of domain at the beginning.

The main step is selecting an appropriate numerical solver. As mentioned before, there are two regimes of flow, that is, laminar and turbulent. In laminar flow, the layers are parallel without any disruption, lateral mixing, and 
eddies [55]. Therefore, the discretized governing equation, that is, continuity, momentum, and energy, can be computed in coarse grid.

On the other hand, the turbulent flow is completely a complex regime with disruption, lateral mixing, and eddies. The implementation of discretized governing equation in turbulent flow needs very fine grid, and so unacceptable computational cost. This method is called Direct Numerical Simulation (DNS). DNS is not an applicable method in real situation. Alternatively, some simplified methods were developed in order to model the turbulent flow and reduce considerably the computational cost, such as Reynolds-Averaged NavierStokes (RANS). The comprehensive description of different methods of turbulent flow simulation is presented by Andersson et al. [56].

\section{Discussion}

The critical point in virtual simulation is the accuracy of prediction and consistency with the reality. The accuracy depends on the variety of factors. In the current paper, the important related subjects are reviewed in a classified manner. Although, different $1 \mathrm{D}$ and 3D simulations of CoW and cerebral aneurysm were carried out, many questions have been left. Some of important objectives of future studies are listed here.

(1) The cerebral perfusion is a vital subject and it is a function of flow rate and pressure. The amount of flow rate is known in normal condition and also the effect of some anomalies on flow rate was studied by $1 \mathrm{D}$ simulation [25]. However, the effect of anomalies and also 3D complexity is not completely obvious.

(2) The $0 \mathrm{D}$ or $1 \mathrm{D}$ modeling can help generate boundary conditions for $3 \mathrm{D}$ simulation. In addition, the $3 \mathrm{D}$ simulation can help improve the $1 \mathrm{D}$ modeling. As a result, $0 \mathrm{D}$ or $1 \mathrm{D}$ models and 3D simulation can have an effect on each other, simultaneously. It means that the local 3D simulation, that is, CoW, and global modeling, that is, cerebral blood system, should perform in a coupled condition. Regarding anomalies influence on the functionalities of CoW and also whole cerebral blood supply system, what is the relation between anomalies and cerebral blood supply?

\section{Disclosure}

There is no financial interest related to the material in the paper.

\section{Conflict of Interests}

The authors declare that there is no conflict of interests regarding the publication of this paper.

\section{Authors' Contribution}

Study concept and design was made by S. R. Ghodsi, V. Esfahanian, and S. M. Ghodsi. Drafting of the paper was made by S. R. Ghodsi. The sponsor had no role in the design and conduct of the study; collection, management, and analysis of the data; or preparation, review, and approval of the paper.

\section{Acknowledgments}

This study was carried out in VFE Research Institute in University of Tehran and based on a Project entitled as "3D Numerical Simulation of Flow in the Circle of Willis in Normal and Common Anomalies." In addition, many thanks to Sina Trauma Research Center, because of spiritual and financial sponsorship of the project.

\section{References}

[1] R. G. Whitmore, R. A. Grant, P. LeRoux, O. El-Falaki, and S. C. Stein, "How large is the typical subarachnoid hemorrhage? A review of current neurosurgical knowledge," World Neurosurgery, vol. 77, pp. 686-697, 2012.

[2] A. W. J. Hoksbergen, B. Fülesdi, D. A. Legemate, and L. Csiba, "Collateral configuration of the circle of Willis: transcranial color-coded duplex ultrasonography and comparison with postmortem anatomy," Stroke, vol. 31, no. 6, pp. 1346-1351, 2000.

[3] M. A. Neimark, A.-A. Konstas, A. F. Laine, and J. Pile-Spellman, "Integration of jugular venous return and circle of Willis in a theoretical human model of selective brain cooling," Journal of Applied Physiology, vol. 103, no. 5, pp. 1837-1847, 2007.

[4] X.-Q. Cheng, J.-M. Tian, C.-J. Zuo, J. Liu, Q. Zhang, and G.M. Lu, "Quantitative perfusion computed tomography measurements of cerebral hemodynamics: correlation with digital subtraction angiography identified primary and secondary cerebral collaterals in internal carotid artery occlusive disease," European Journal of Radiology, vol. 81, pp. 1224-1230, 2012.

[5] K. R. D. De Silva, R. Silva, D. Amaratunga, W. Gunasekera, and R. W. Jayesekera, "Types of the cerebral arterial circle (circle of Willis) in a Sri Lankan population," BMC Neurology, vol. 11, article 5, 2011.

[6] V. Papantchev, S. Hristov, D. Todorova et al., "Some variations of the circle of Willis, important for cerebral protection in aortic surgery-a study in Eastern Europeans," European Journal of Cardio-thoracic Surgery, vol. 31, no. 6, pp. 982-989, 2007.

[7] B. Eftekhar, M. Dadmehr, S. Ansari, M. Ghodsi, B. Nazparvar, and E. Ketabchi, "Are the distributions of variations of circle of Willis different in different populations?-results of an anatomical study and review of literature," BMC Neurology, vol. 6, article 22, 2006.

[8] M. A. Maaly and A. A. Ismail, "Three dimensional magnetic resonance angiography of the circle of Willis: anatomical variations in general Egyptian population," Egyptian Journal of Radiology and Nuclear Medicine, vol. 42, no. 3-4, pp. 405-412, 2011.

[9] J. Ottesen, M. Olufsen, and J. Larsen, Applied Mathematical Models in Human Physiology, Department of Mathematics and Physics, Roskilde University, 2006.

[10] J. Janzen, "Ageing of the conduit arteries," Journal of Pathology, vol. 211, pp. 157-172, 2007.

[11] R. Uflacker and C. J. Feldman, Atlas of Vascular Anatomy, Williams \& Wilkins, 1997.

[12] I. Chatziprodromou, A. Tricoli, D. Poulikakos, and Y. Ventikos, "Haemodynamics and wall remodelling of a growing cerebral aneurysm: a computational model," Journal of Biomechanics, vol. 40, no. 2, pp. 412-426, 2007. 
[13] K. Rogers, Blood: Physiology and Circulation, Britannica Educational Publishing, 2012.

[14] http://www.cvphysiology.com/Hemodynamics/H007.htm.

[15] K. Valen-Sendstad, K.-A. Mardal, M. Mortensen, B. A. P. Reif, and H. P. Langtangen, "Direct numerical simulation of transitional flow in a patient-specific intracranial aneurysm," Journal of Biomechanics, vol. 44, no. 16, pp. 2826-2832, 2011.

[16] F. N. van De Vosse and N. Stergiopulos, "Pulse wave propagation in the arterial tree," Annual Review of Fluid Mechanics, vol. 43, pp. 467-499, 2011.

[17] F. Yilmaz and M. Y. Gundogdu, "A critical review on blood flow in large arteries; relevance to blood rheology, viscosity models, and physiologic conditions," Korea Australia Rheology Journal, vol. 20, no. 4, pp. 197-211, 2008.

[18] Ø. Evju, K. Valen-Sendstad, and K. A. Mardal, "A study of wall shear stress in 12 aneurysms with respect to different viscosity models and flow conditions," Journal of Biomechanics, vol. 46, pp. 2802-2808, 2013.

[19] C. Caro, The Mechanics of the Circulation, Cambridge University Press, 2012.

[20] Y. Huo and G. S. Kassab, "Pulsatile blood flow in the entire coronary arterial tree: theory and experiment," The American Journal of Physiology-Heart and Circulatory Physiology, vol. 291, no. 3, pp. H1074-H1087, 2006.

[21] Y. Fung, Biomechanics: Circulation, Springer, 1996.

[22] http://hypertextbook.com/facts/2004/MichaelShmukler.shtml.

[23] H. White and B. Venkatesh, "Cerebral perfusion pressure in neurotrauma: a review," Anesthesia and Analgesia, vol. 107, no. 3, pp. 979-988, 2008.

[24] S. Moore, T. David, J. G. Chase, J. Arnold, and J. Fink, “3D models of blood flow in the cerebral vasculature," Journal of Biomechanics, vol. 39, no. 8, pp. 1454-1463, 2006.

[25] J. Alastruey, K. H. Parker, J. Peiró, S. M. Byrd, and S. J. Sherwin, "Modelling the circle of Willis to assess the effects of anatomical variations and occlusions on cerebral flows," Journal of Biomechanics, vol. 40, no. 8, pp. 1794-1805, 2007.

[26] P. Reymond, Y. Bohraus, F. Perren, F. Lazeyras, and N. Stergiopulos, "Validation of a patient-specific one-dimensional model of the systemic arterial tree," The American Journal of PhysiologyHeart and Circulatory Physiology, vol. 301, no. 3, pp. H1173H1182, 2011.

[27] D. M. Sforza, C. M. Putman, and J. R. Cebral, "Computational fluid dynamics in brain aneurysms," International Journal for Numerical Methods in Biomedical Engineering, vol. 28, pp. 801808, 2012.

[28] A. Quarteroni and A. Veneziani, "Analysis of a geometrical multiscale model based on the coupling of ODE and PDE for blood flow simulations," Multiscale Modeling \& Simulation, vol. 1, pp. 173-195, 2003.

[29] B. W. A. M. M. Beulen, M. C. M. Rutten, and F. N. van de Vosse, "A time-periodic approach for fluid-structure interaction in distensible vessels," Journal of Fluids and Structures, vol. 25, no. 5, pp. 954-966, 2009.

[30] G. Austin, "Biomathematical model of aneurysm of the circle of willis, I: the duffing equation and some approximate solutions," Mathematical Biosciences, vol. 11, no. 1-2, pp. 163-172, 1971.

[31] J. Cronin, "Mathematical model of aneurysm of the circle of Willis: II. A qualitative analysis of the equation of Austin," Mathematical Biosciences, vol. 22, pp. 237-275, 1974.

[32] F. Cassot, M. Zagzoule, and J.-P. Marc-Vergnes, "Hemodynamic role of the circle of Willis in stenoses of internal carotid arteries. An analytical solution of a linear model," Journal of Biomechanics, vol. 33, no. 4, pp. 395-405, 2000.

[33] S. Lorthois, F. Cassot, and F. Lauwers, "Simulation study of brain blood flow regulation by intra-cortical arterioles in an anatomically accurate large human vascular network. Part II: flow variations induced by global or localized modifications of arteriolar diameters," NeuroImage, vol. 54, no. 4, pp. 2840-2853, 2011.

[34] S. Lorthois, F. Cassot, and F. Lauwers, "Simulation study of brain blood flow regulation by intra-cortical arterioles in an anatomically accurate large human vascular network: part I: methodology and baseline flow," NeuroImage, vol. 54, no. 2, pp. 1031-1042, 2011.

[35] J. P. Mynard and P. Nithiarasu, "A 1D arterial blood flow model incorporating ventricular pressure, aortic vaive ana regional coronary flow using the locally conservative Galerkin (LCG) method," Communications in Numerical Methods in Engineering, vol. 24, no. 5, pp. 367-417, 2008.

[36] J. R. Cebral, F. Mut, J. Weir, and C. M. Putman, "Association of hemodynamic characteristics and cerebral aneurysm rupture," American Journal of Neuroradiology, vol. 32, no. 2, pp. 264-270, 2011.

[37] J. Xiang, S. K. Natarajan, M. Tremmel et al., "Hemodynamicmorphologic discriminants for intracranial aneurysm rupture," Stroke, vol. 42, no. 1, pp. 144-152, 2011.

[38] Y. Qian, H. Takao, M. Umezu, and Y. Murayama, "Risk analysis of unruptured aneurysms using computational fluid dynamics technology: preliminary results," American Journal of Neuroradiology, vol. 32, no. 10, pp. 1948-1955, 2011.

[39] Y. Miura, F. Ishida, Y. Umeda et al., "Low wall shear stress is independently associated with the rupture status of middle cerebral artery aneurysms," Stroke, vol. 44, pp. 519-521, 2013.

[40] H. Takao, Y. Murayama, S. Otsuka et al., "Hemodynamic differences between unruptured and ruptured intracranial aneurysms during observation," Stroke, vol. 43, no. 5, pp. 14361439, 2012.

[41] J. G. Isaksen, Y. Bazilevs, T. Kvamsdal et al., "Determination of wall tension in cerebral artery aneurysms by numerical simulation," Stroke, vol. 39, no. 12, pp. 3172-3178, 2008.

[42] A. I. Barakat, "Blood flow and arterial endothelial dysfunction: mechanisms and implications," Comptes Rendus Physique, vol. 14, no. 6, pp. 479-496, 2013.

[43] R. Torii, M. Oshima, T. Kobayashi, K. Takagi, and T. E. Tezduyar, "Fluid-structure interaction modeling of blood flow and cerebral aneurysm: significance of artery and aneurysm shapes," Computer Methods in Applied Mechanics and Engineering, vol. 198, no. 45-46, pp. 3613-3621, 2009.

[44] K. Yokoi, F. Xiao, H. Liu, and K. Fukasaku, “Three-dimensional numerical simulation of flows with complex geometries in a regular Cartesian grid and its application to blood flow in cerebral artery with multiple aneurysms," Journal of Computational Physics, vol. 202, no. 1, pp. 1-19, 2005.

[45] C. A. Figueroa, I. E. Vignon-Clementel, K. E. Jansen, T. J. R. Hughes, and C. A. Taylor, "A coupled momentum method for modeling blood flow in three-dimensional deformable arteries," Computer Methods in Applied Mechanics and Engineering, vol. 195, no. 41-43, pp. 5685-5706, 2006.

[46] E. S. Di Martino, G. Guadagni, A. Fumero et al., "Fluidstructure interaction within realistic three-dimensional models of the aneurysmatic aorta as a guidance to assess the risk of rupture of the aneurysm," Medical Engineering and Physics, vol. 23, no. 9, pp. 647-655, 2001. 
[47] Z. Li and C. Kleinstreuer, "A comparison between different asymmetric abdominal aortic aneurysm morphologies employing computational fluid-structure interaction analysis," European Journal of Mechanics B, vol. 26, no. 5, pp. 615-631, 2007.

[48] D. I. Zuleger, D. Poulikakos, A. Valavanis, and S. S. Kollias, "Combining magnetic resonance measurements with numerical simulations-extracting blood flow physiology information relevant to the investigation of intracranial aneurysms in the circle of Willis," International Journal of Heat and Fluid Flow, vol. 31, no. 6, pp. 1032-1039, 2010.

[49] T. David and S. Moore, "Modeling perfusion in the cerebral vasculature," Medical Engineering and Physics, vol. 30, no. 10, pp. 1227-1245, 2008.

[50] L. Grinberg, T. Anor, E. Cheever, J. R. Madsen, and G. E. Karniadakis, "Simulation of the human intracranial arterial tree," Philosophical Transactions of the Royal Society A, vol. 367, no. 1896, pp. 2371-2386, 2009.

[51] Q. Long, L. Luppi, C. S. König, V. Rinaldo, and S. K. Das, "Study of the collateral capacity of the circle of Willis of patients with severe carotid artery stenosis by $3 \mathrm{D}$ computational modeling," Journal of Biomechanics, vol. 41, no. 12, pp. 2735-2742, 2008.

[52] M. S. Alnæs, J. Isaksen, K.-A. Mardal, B. Romner, M. K. Morgan, and T. Ingebrigtsen, "Computation of hemodynamics in the circle of Willis," Stroke, vol. 38, no. 9, pp. 2500-2505, 2007.

[53] C. S. Kim, "Numerical simulation of auto-regulation and collateral circulation in the human brain," Journal of Mechanical Science and Technology, vol. 21, no. 3, pp. 525-535, 2007.

[54] http://www.materialise.com/.

[55] G. Batchelor, An Introduction to Fluid Dynamics, Cambridge University Press, 2000.

[56] B. Andersson, R. Andersson, L. Håkansso, M. Mortensen, R. Sudiyo, and B. van Wachem, Computational Fluid Dynamics for Engineers, Cambridge University Press, 2012. 


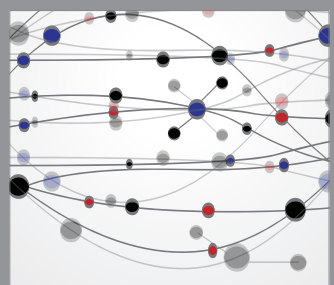

The Scientific World Journal
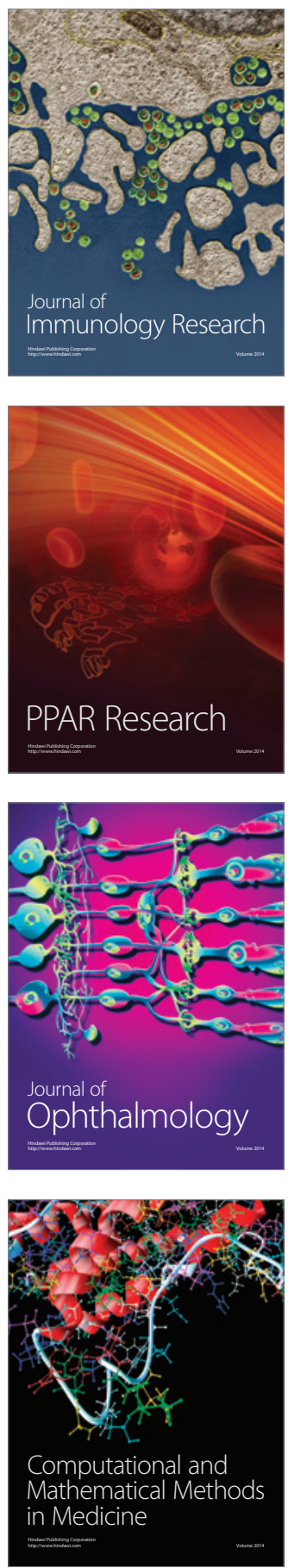

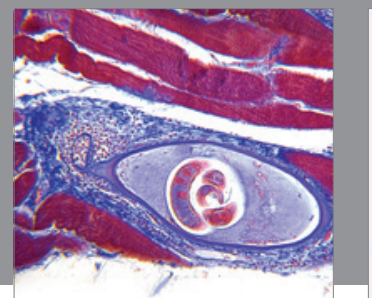

Gastroenterology

Research and Practice
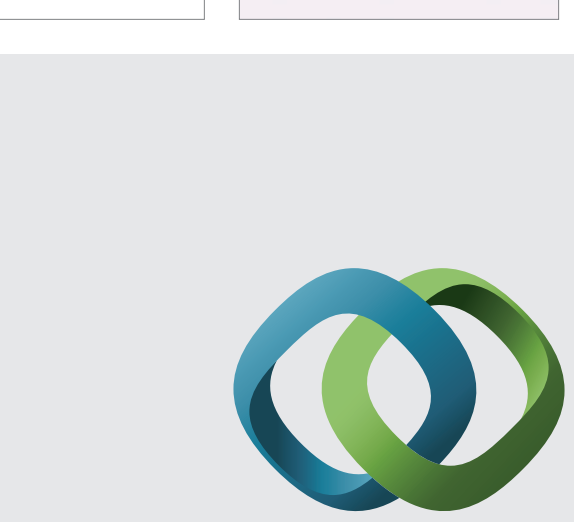

\section{Hindawi}

Submit your manuscripts at

http://www.hindawi.com
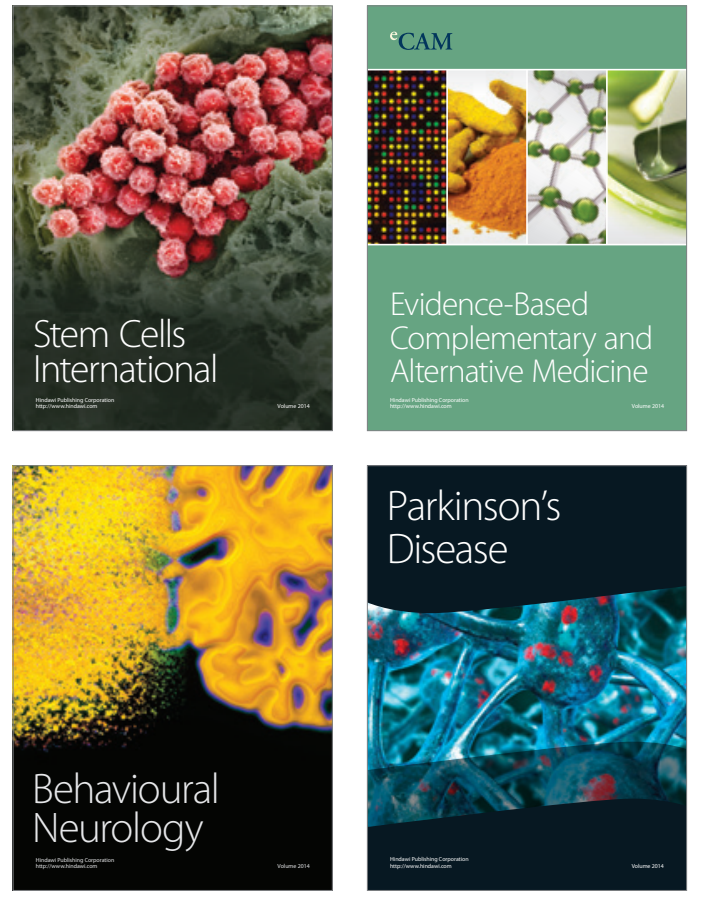
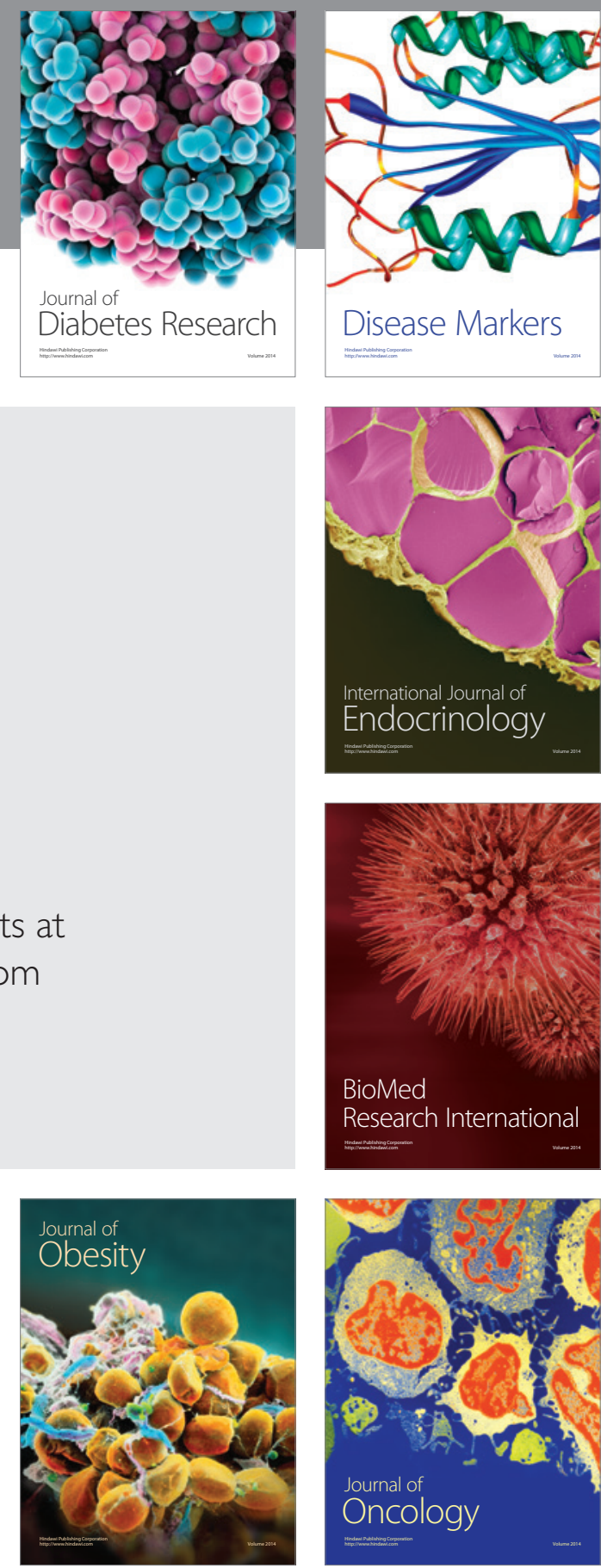

Disease Markers
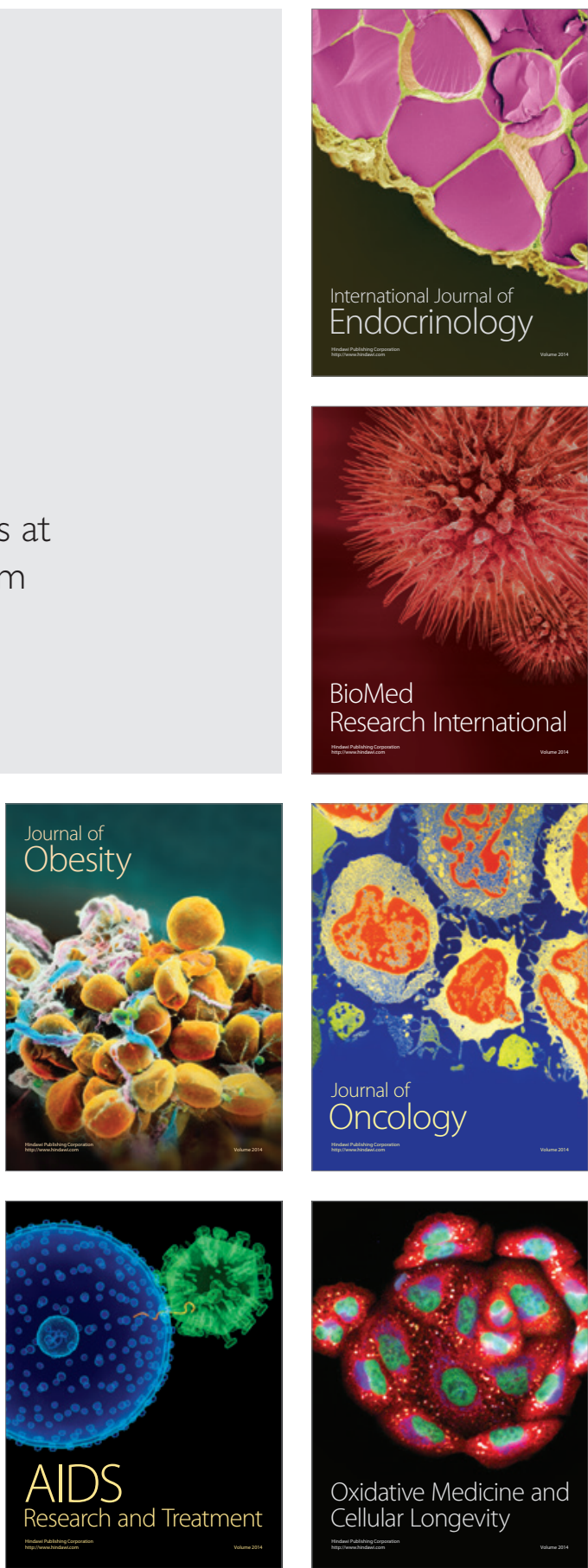